\title{
Fish bone ingestion in a toddler
}

\author{
Dr. Surinder K Singhal, Dr.Ankit Gulati, Dr. Nitin Gupta, Dr. Devender Dayal, Dr. Vinci Priyasha
}

Dept of ENT, GMCH-32, Chandigarh.

${ }^{*}$ Corresponding author

Dr. Ankit Gulati (MS,DNB), Dept of ENT, GMCH-32, Chandigarh.

Submitted: 16 May2020; Accepted:22 May2020; Published: 27 May 2020

\section{Introduction}

Ingestion of foreign body is one of the most common problems among children.It is very commonly seen between the ages of 6 months and 6 years after accidental ingestion during playtime, this being the stage of exploratory development, which may lead to serious complications. There are various presentations of patients with foreign body ingestion, starting from vomiting, a sensation of something being stuck, odynophagia, and dysphagia, etc. Apart from having symptoms, some patients or their parents may state a history of ingestion only. Most common foreign body ingestion seen in children are the coins followed by button battery cells [1].

\section{Case Summary}

A 1.5-year-old child was brought to the ENT emergency with his mother giving the history of ingestion of fishbone while having dinner. As the child was 1.5 years old and was hardly able to speak, there was no other way of knowing the exact history except for going on the word of his mother.There were no major symptoms apart from the history of drooling of saliva post-ingestion at home as per the history given bythe mother. There was no history of any vomiting or any blood in vomiting or sputum. No history of any difficulty in breathing or swallowing. The child presented in a relatively healthy state, however the child was pointing his fingers towards his neck giving indications of something being stuck in the throat.On general physical examination, the child was apparently normal and was showing no signs of any distress. On further local examination, in the oral cavity inspection; there were primary teeth with both upper and lower incisors, tonsillar fossa normal, posterior pharyngeal wall was normal. On palpation, there was no tenderness or any evidence of fishbone or any other foreign body in the oral cavity. On palpation of the base of tongue; there was no evidence of any lodgement of foreign body in the base of the tongue and adjacent areas. On inspection of the neck, there was no abnormality detected, on palpation; laryngeal contour was normal, no palpable lymph nodes or any point of tenderness present.

Further investigations were carried out on suspicion. For radiological investigation i.e. an X-ray of soft tissue neck and chest was done (Figure1). But to our astonishment, even the X-ray films also turned out to be normal, showing no signs or any evidence of any foreign body.
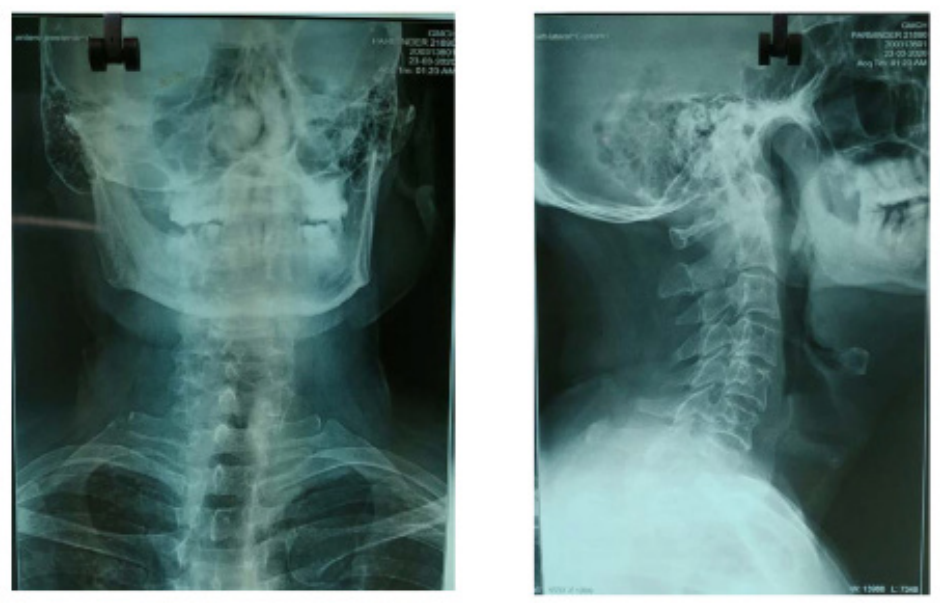

Figure 1 - X-Ray STN Anteroposterior and lateral view

The child was admitted in the emergency and further plan of action was decided, which was to opt for diagnostic endoscopy of larynx and oesophagus and proceed. The consent was taken from the parents and the child was taken up for emergency OT. The child was shifted on the OT table and was induced under general anaesthesia. The paediatric sized rigid direct laryngoscope was introduced, and the larynx was examined but there was no evidence of any foreign body found in the larynx. The direct laryngoscope was removed. Then, the rigid hypopharyngoscope was introduced carefully visualizing all the structures. And eventually, there was a fishbone, found lodged in the right pyriform sinus.

The foreign body was visualized and was carefully removed. It was around $1.5 \mathrm{~cm}$ long fishbone (Figure 2), secured and then kept in a specimen container. All the other spaces in the pharynx were carefully visualized, the hypopharyngoscope was removed meticulously envisioning all the structures and looking for any trauma due to the scope. A paediatric oesophagoscope was introduced and esophagoscopy was done to rule out any other possible lodgement of other foreign bodies. There was no evidence of any other foreign body found in the oesophagus, or anywhere in the upper respiratory and digestive tract. The oesophagoscope was removed gently, visualizing all the structures in the reverse order 
along with suctioning of the secretions making sure no trauma due to the scope was done. Ryles tube was put in n secured. Ryles tube was removed after 24 hours. The child was then handed over to the anaesthetist and reversal of anaesthesia was done following which the child was shifted to the recovery room for post-operative care in stable condition. The post-operative period was uneventful and then the child was shifted back to the ward. The parents of the child were overwhelmed to see the child well and offered gratitude for removing the fishbone. The child recovered well and faced no postoperative complications and was discharged after 3 days.

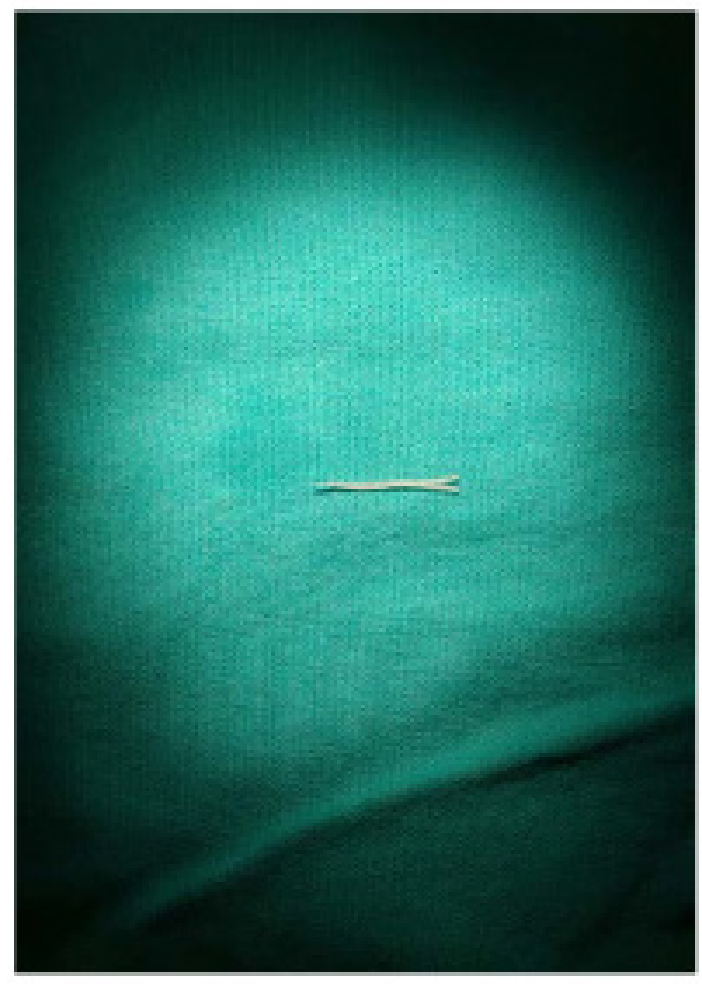

Figure 2 - Fish bone

\section{Discussion}

Fish bone foreign body (FFB) is one of the most commonly ingested foreign bodies (FBs) encountered in the emergency, accounting for 46 to $88 \%$ of the total adult $\mathrm{FB}$ admissions [3]. It is themost common FB across Asia, the Mediterranean, and other coastal countries, assumingly due to their dietary habits [3].They are common in the paediatric population, though the estimated prevalence is lower than in adults, estimated at around 30\% [4].Most fish bones are usually identified within the oral cavity or oropharynx, reinforcing the significance of thorough oral examination.1The most common locations for fish bones discovered are mainly in the palatine tonsils, base of the tongue, valleculae, and the pyriform sinus [5].The foreign body lodged could lead to complications includingesophageal perforation and result in catastrophic consequences such as deep neck infection, mediastinitis, or even aortoesophagealfistula. Othercomplications associated with FB are vascular manifestations, such as massive haemorrhage or a pulsatile cervical mass [1].Plain radiography is the most common investigative study performed in patients presenting with suspected fish-bone foreign body impaction. However, $48 \%$ of fish bones are radiolucent, and diagnostic accuracy is low because fish bones are consistent with the varying radiodensity of cartilage and bones [6].
Fish bones lodged in the upper aerodigestive tract may be dislodged, and removed by direct visualization or through endoscopy, and in some of the cases, lodged bones pass through the gastrointestinal tract. Foreign bodies such as fish bones can be removed via flexible endoscopy in adults, however, in children, general anesthesia and rigid laryngoesophagoscopy are the standard of care [7].Although the need for open exploration in pediatric patients with FB ingestion is only necessary in $1 \%$ of the total cases, sharp FB cause a more frequent rate of complications (between 15 and 30\%) [1].In our case the history and clinical picture of the patient was suggestive of foreign body ingestion which being a fish bone according to the history. However, on radiological investigation, there was no evidence of any foreign body. It was a tough decision to opt for diagnostic rigid endoscopy, as the patient was a 1.5-year-old child, but it proved out to be a right one. And we found a fish bone found lodged in the right pyriform sinus in the hypopharynx on endoscopy. Fish bone ingestion in a 1.5-year-old patient is a very rare presentation and with a successful removal without any complications is something to be documented.

\section{References}

1. Marsha K, Robert W.Foreign (2013) body ingestions in the pediatric population and techniques of endoscopic removal. Tech Gastrointest Endosc15:9-17.

2. Khorana J, Tantivit Y, Phiuphong C, Pattapong S, Siripan S, et al. (2019) Foreign Body Ingestion in Pediatrics: Distribution, Management and Complications. Medicina 55: 1-13.

3. Klein A, Ovnat-Tamir S, Marom T, Gluck O, Rabinovics N, et al. (2019) Fish bone foreign body: The role of imaging.Int $\mathbf{J}$ Otorhinolaryngol Head Neck Surg 23:110-115.

4. Higo R, Matsumoto Y, Ichimura K, Kaga K (2003) Foreign bodies in the aerodigestive tract in pediatric patients. Auris Nasus Larynx 30:397-401.

5. Kim JP, Kwon OJ, Shim HS, Kim RB, Kim JH, et al. (2015) Analysis of clinical feature and management of fish bone ingestion of upper gastrointestinal tract.Clin Exp Otorhinolaryngol8:261-267.

6. Woo SH, Kim KH (2015) Proposal for methods of diagnosis of fish bone foreign body in the esophagus. Laryngoscope 125:2472-2475.

7. Goldman RD, Gurberg J, Moxham JP (2018) A Lodged Barramundi Fish-Bone Stabbing the Piriform Fossa in a Child. PediatrEmerg Care34:243-245.

Copyright: (C2020 Dr.Ankit Gulati. This is an open-access article distributed under the terms of the Creative Commons Attribution License, which permits unrestricted use, distribution, and reproduction in any medium, provided the original author and source are credited. 